\title{
Hipersensibilidade dentinária em lesões cervicais não cariosas: etiologia e tratamento
}

Dentin hypersensitivity in non-carious cervical lesions: etiology and treatment

Hipersensibilidad dentinaria en lesiones cervicales no carias: etiología y tratamiento

Bruna Caroline REGIANI ${ }^{\mathbf{1}}$
Heloise Nogueira ROCHA
Valdinéia Maria TOGNETTI
Alessandra Pereira de ANDRADE

Alessandra Pereira de ANDRADE

${ }^{I}$ Curso de Odontologia da Universidade São Francisco- USF/ campus Bragança Paulista- SP

${ }^{2}$ Mestre do Curso de Odontologia da Universidade São Francisco-USF/ campus Bragança Paulista- SP

${ }^{3}$ Doutora do Curso de Odontologia da Universidade São Francisco-USF/ campus Bragança Paulista-SP

\section{Resumo}

A hipersensibilidade dentinária é uma temática muito presente a pratica diária do cirurgião-dentista; apesar de estudos aplicados frente à estimativa e prevalência mostrarem-se de bastante valia, há grande dificuldade na obtenção de dados científicos pela subjetividade da sintomatologia. Objetivando ampliar o conhecimento de acadêmicos e profissionais da área de Odontologia quanto a etiologia e possíveis atuais tratamentos através de levantamento de referências sobre o tema, esta breve revisão da literatura, que utilizou as bases de dados : Bireme, PUBmed, ResearchGate e Scielo; tem como finalidade relacionar a hipersensibilidade dentinária cervical com as lesões cervicais não cariosas; sendo elas: abrasão, abfração, atrição e erosão.

Descritores: Sensibilidade da Dentina; Dentina; Diagnóstico; Terapêutica.

\section{Abstract}

Dentin hypersensitivity is a very present theme in the daily practice of the dental surgeon; although studies applied in the face of estimation and prevalence prove to be quite valuable, there is great difficulty in obtaining scientific data through the subjectivity of the symptomatology. This brief literature review, which used the databases: Bireme, PUBmed, ResearchGate and Scielo, aims to relate cervical dentin hypersensitivity with non-carious cervical lesions; being: abrasion, abfraction, attrition and erosion. Aiming to broaden the knowledge of dental practitioners and professionals regarding the etiology and possible current treatments it was a survey of references on the topic in the last twenty years.

Descriptors: Dentin Sensitivity; Dentin; Diagnosis; Therapeutics.

\section{Resumen}

La hipersensibilidad a la dentina es un tema muy presente en la práctica diaria del dentista; Aunque los estudios aplicados a la estimación y la prevalencia demuestran ser muy valiosos, existe una gran dificultad para obtener datos científicos debido a la subjetividad de los síntomas. Con el objetivo de ampliar el conocimiento de los académicos y profesionales dentales sobre la etiología y los posibles tratamientos actuales a través de una encuesta de referencias sobre el tema, esta breve revisión de literatura, que utilizó las bases de datos Bireme, PUBmed, ResearchGate y Scielo; pretende relacionar la hipersensibilidad de la dentina cervical con lesiones cervicales no cariosas; a saber: abrasión, abfracción, desgaste y erosión

Descriptores: Sensibilidad de la Dentina; Dentina; Diagnóstico; Terapéutica.

\section{INTRODUÇÃO}

A hipersensibilidade dentinária (HD), pode ser definida como o desconforto gerado frente a perda do material de proteção, o esmalte dental, e exposição dos túbulos dentinários devido a fatores químicos e mecânicos de baixa intensidade e alta frequência, sendo estes adquiridos, portanto não podendo ser associados a nenhuma outra patologia dental. Deve-se ressaltar que sensibilidade e hipersensibilidade são conceitos diferentes. Sensibilidade dentinária caracteriza-se pela resposta normal do individuo frente a um estimulo sensitivo, já a hipersensibilidade está relacionada a uma ótica exacerbada desta resposta tendo causas multifatoriais ${ }^{1,2}$.

Um dos assuntos mais comumente relacionados a esta alteração são as lesões cervicais não-cariosas (LCNCs).Que referem-se à perda de tecido mineralizado na região cervical da coroa dentária e superfície radicular subjacente por meio de um processo não relacionado à cárie. Essas lesões são comumente classificadas em: abrasão, abfração, atrição e erosão. Portanto, o reconhecimento e a identificação dos fatores de risco são de grande valia para diagnóstico, prevenção e tratamento, evitando a evolução do quadro ou o aparecimento de novas $\mathrm{LCNCs}^{3}$.

A dentina e a polpa são tecidos que estão interligados tanto estruturalmente quanto funcionalmente, sendo estes ambos originados na odontogênese através da papila dentária. Sendo assim, as células da polpa, os odontoblastos, são responsáveis pela síntese e deposição da matriz de dentina, e, portanto permanecem com seus prolongamentos citoplasmáticos no interior dos túbulos dentinários ${ }^{4}$. A teoria hidrodinâmica de Brännström, a mais aceita atualmente para explicar o fenômeno de hipersensibilidade dentinária, explica que a decorrência da perturbação de fluidos presentes no interior dos túbulos tanto no sentido da polpa como o inverso, resultante de estímulos térmicos, evaporativos, táteis, osmóticos ou químicos, quando aplicados sobre a dentina exposta, desencadeia a distensão ou contração dos prolongamento odontoblásticos ,ativando assim as ramificações nervosas presentes no início do prolongamento dessas células, e acarretando na sensação dolorosa; sendo assim quando estes cessam, cessa também a sintomatologia de acordo com o limiar de sensibilidade ${ }^{2,5,6}$.

A história prévia da hipersensibilidade bem como a correlação de fatores como presença de LCNCs e predisposições a hábitos que afetem na sintomatologia, direcionarão o profissional ao correto diagnostico, planejamento e tratamento. Atualmente 
no cenário geral, os tratamentos buscam a obliteração dos túbulos dentinários. Porém há um grande desafio no emprego destes produtos, em questões como biocompatibilidade e durabilidade. As informações sobre a prevalência da HD mostram-se dependentes de fatores para obtenção dos dados fonte como: população de amostra (origem étnica, ambiente, avaliação periodontal, cuidados com a saúde bucal); e critério utilizado para avaliação do paciente; clinicamente, pelo profissional, ou pelo próprio paciente por meio de questionários ${ }^{7}$.

Estudos de 2012 realizados com a população brasileira, na região de São Paulo, estimaram que a prevalência da HD era de 46\%; sendo as mulheres mais atingidas, por apresentarem melhor relação de higiene bucal que os homens. Estes dados se contrastam com os primeiros estudos realizados por Fischer em 1992, diagnosticando apenas 17\% neste âmbito; apesar de se tratarem de populações diferentes, evidencia-se que com aumento da expectativa de vida, e o maior numero de indivíduos com dentes naturais ao longo da vida, a resultante deste fato corroboram para o aumento da prevalência demonstrado ${ }^{8,9}$.

A proposta revisão bibliográfica dos últimos vinte anos, visa contribuir para ampliação do conhecimento a cerca da HD, correlacionada as LCNCs, que se mostram cada vez mais presente na clinica diária. Com o aumento da expectativa de vida e do número de pessoas que expõem seus dentes em maior período de tempo aos fatores etiológicos, como mudanças culturais e hábitos parafuncionais; o correto diagnóstico e seus possíveis diferenciais dependem diretamente da experiência e o conhecimento do profissional para que se direcione corretamente aos possíveis tratamentos. Objetivando assim ampliar o conhecimento de acadêmicos e profissionais da área de Odontologia quanto a etiologia e possíveis atuais tratamentos.

\section{MATERIAL E MÉTODO}

Esta breve revisão bibliográfica, utilizou as bases de dados : Bireme, PUBmed, ResearchGate e Scielo; aplicando os seguintes descritores de busca em português "hipersensibilidade" "lesões cervicais não cariosas", em inglês "hypersensitivity", "lession non-carious". Como critério de inclusão foram considerados aqueles que continham os dois temas associados.Foram utilizados artigos de revisão de literatura, revisão sistemática, estudos clínicos randomizados, bem como capitulo de livros pertinentes aos dois temas, publicados no período de 1992 e 2018.

\section{RESULTADOS}

- Lesões cervicais não cariosas e Teoria de Brännström: definição, etiologia e fatores relacionados
Morfologicamente em condições clínicas normais, o esmalte dentário e o cimento protegem os tecidos tubulares dentinários impedindo-os de serem expostos ao meio bucal. Portanto quando algum processo remove esta proteção, criam-se então condições clínicas para o aparecimento da sintomatologia da hipersensibilidade dentinária cervical. Estes processos atualmente são chamados por alguns autores de alterações regressivas dentais, podendo estes serem resultado de ação de agentes injuriosos ,e não podendo ser associadas a anomalias de desenvolvimento ou como lesões inflamatórias. E sobre estes processos aqui descritos correspondem a eles os nomes de: abfração, abrasão, atrição e erosão ${ }^{10,11}$.

Tanto fatores intrínsecos como extrínsecos podem estar relacionados a estas alterações de estruturas mineralizadas, sendo estes aplicados em conjunto e mutuamente deixando os dentes susceptíveis a ocorrência de sistemas de ordem fisiológica ${ }^{2,5-6}$. A dor provocada, leve e moderada, que desaparece quando retirado o agente causador, os estímulos para movimentação dos fluidos, não deve ser confundida com patologias relacionadas às doenças pulpares de origem endodôntica, sendo que estas não são decorrentes de estímulos transitórios e não cessam quando estes são removidos ${ }^{10}$.

- Abfração

Denomina-se abfração, toda perda de estrutura dental calcificada, advindo de repetida pressão, causada por estresse de forças oclusais. Porém em sua etiologia há uma ocorrência de complexa interação entre fatores de origem química e mecânica. As concentrações de esforços mastigatórios aplicados excentricamente através de forças oclusais de tração ou cisalhamento que não se distribuem na direção do longo eixo do dente ao dente, resultam na inclinação dental que rompem as ligações químicas das estruturas calcificadas, criando microfraturas ou trincas que através das quais proporcionaram a entrada de moléculas de saliva e água, tornando a região mais susceptível a efeito de solubilização de ácidos e remoção durante a escovação; formando lesão bem definida em forma de cunha na face vestibular, geralmente profunda e na superfície vestibular; sendo mais comum na região cervical dos dentes ${ }^{6,10-12}$.

A maior incidência de lesão de abfração é observada respectivamente nos pré-molares superiores, onde se concentra maior força mastigatória; pré-molares inferiores, caninos e em menor incidência nos incisivos inferiores. Portanto a abfração acomete dentes periodontalmente sadios, hígidos, e sem mobilidade devido a necessidade da condição para criação da tensão. Desta forma, com a maior rigidez do periodonto, os pacientes mais idosos podem ter maior acometimento pela lesão de abfração, já que a estrutura de suporte perde parte da 
capacidade de absorver forças oclusais. Porém é importante ressaltar que nem toda lesão irá gerar resposta dolorosa de $\mathrm{HD}$; neste caso com o próprio avanço da idade, atresiamento da polpa e consequentemente de seus prolongamentos, a formação de dentina esclerótica acaba por produzir a impermeabilização a região ${ }^{6,10-13}$.

\section{- Abrasão}

A abrasão dental caracteriza-se pelo desgaste da estrutura dental sadia. Neste caso o desgaste não é correlacionado as forças oclusais. Ocorre quando uma superfície áspera e dura desliza sobre outra superfície com o modulo menor de rigidez criando-se assim ranhuras. Portanto há uma perda de substância calcificada pela ação de processos mecânicos repetitivos, podendo estes serem associados a objetos ou substâncias introduzidas na boca ${ }^{10,12}$.

Os principais fatores etiológicos estão relacionados aos procedimentos de higiene bucal; sendo estes: a escovação inadequada, e/ou emprego de escova incorreta; bem como a utilização excessiva de agentes abrasivos contidos nos dentifrícios e afins. Atualmente existem vários tipo de agentes abrasivos contidos nos dentifrícios comercializados sendo estes: Carbonato de Cálcio (27\%), Sílica (26\%), Bicarbonato de Sódio, Dióxido de Silício, Dióxido de Titânio, Fosfato Trissódico, Óxido de Alumínio e Silicato de Sódio, todos com a proposta de prevenir o manchamento dental ${ }^{14}$.

Com a mudança dos hábitos culturais e conceito de beleza, cada vez mais há esta procura por produtos que prometem estes tipos de resultados imediatos. Porém, a falta de informação nestes produtos, sobre o nível de abrasividade e o tipo de propaganda tendenciosa, corroboram muitas vezes para seu uso não racional, gerando agressões aos dentes e a gengiva. Outros fatores etiológicos citados por alguns trabalhos relacionam a abfração com hábitos nocivos como morder lápis, tampa de caneta, grampo de cabelos entre outros. Identificação do tempo, frequência e a força aplicada durante, devem ser levados em consideração como fatores que podem resultar no aparecimento da lesão ${ }^{6,11,14}$.

Clinicamente as lesões se apresentam região cervical de aspecto duro, altamente polido, de acordo com nível da abrasão; com contornos regulares, rasa e com margens agudas, podendo muitas vezes revelar a coloração da dentina sob fina de camada de esmalte; que pode atingir um único dente ou um grupo deles. Os dentes mais acometidos são os de maior proeminência, sendo estes os caninos e os prémolares ${ }^{12}$.

○ Atrição

A atrição dental corresponde ao desgaste mecânico que afeta as faces incisais e oclusais devido a fatores relacionados a hiperfunção oclusal, e/ou hábitos parafuncionais como apertamento e o briquimismo.O desgaste ocorre quando há aplicação de forças de baixas intensidades, porém constantes, entre dentes antagonistas,o que resulta na aparência de facetas incisivas cada vez mais planas ${ }^{6}$.

A ocorrência é mais prevalente nas faces oclusais, incisais e linguais dos dentes anteriores superiores, e vestibulares dos dentes anteriores inferiores. As primeiras manifestações revelam um pequeno desgaste localizado nestas faces, ou então, um ligeiro aplainamento (achatamento). Quando em fase avançada podem se mostrar facetas dentais em grandes desgastes, planas, lisas, brilhantes deixando a estrutura da dentina exposta ao meio bucal tendo o aspecto de cor amarelada ou castanha, predispondo a possibilidade de estimulação odontoblástica resultando na HD. Devido à relação ao trauma oclusal, mesmo não afetando regiões cervicais diretamente, sua importância se dá na correlação com as lesões de abfração. Considerando que os dentes absorvem impactos, se aplicados em longo eixo, na ocorrência de aplicações destas forças excentricamente em região, as tensões aplicadas sobre as faces incisais podem gerar impacto e influenciar nas lesões em regiões cervicais; portanto quando se da sua ocorrência, esta deve ser diagnosticada e investigada ${ }^{1,6}$.

\section{- Erosão e Biocorrosão}

Diferentes literaturas relacionam o termo erosão com dissolução química da estrutura dentaria provida pela ação de ácidos, sem o envolvimento bacteriano $^{9,10,12}$.

Porém, autores sugerem uma mudança de paradigma em relação a esta definição que falha ao não reconhecer os efeitos de proteólise $\mathrm{e}$ piezoeléctricos. Estudos mais significativos, revisados e integrados indicam que biocorrosão seria a melhor definição para este tipo de lesão, já que aborda a degradação química, bioquímica e eletroquímica da substância dentária causada por ácidos endógenos e exógenos, agentes proteolíticos, bem como os efeitos piezoelétricos apenas na dentina. Portanto, um termo mais preciso que erosão ${ }^{13}$.

O desafio acido percorrido neste tipo de lesão, muitas vezes é apresentado levando em consideração apenas o $\mathrm{pH}$ das estruturas mineralizadas em comparação a dietas ricas em ácidos (fatores extrínsecos). Sendo o esmalte $\mathrm{pH}$ 5,5 e a dentina $\mathrm{pH} 4,5$, é fato que a diferença entre a quantidade de matriz inorgânica entre um e outro irá facilitar a dissolução em maior numero da perda de estrutura de esmalte do que dentina ( matriz que contém mais de $30 \%$ orgânica) quando frente a $\mathrm{pH}$ critico. Porém apenas a concentração de ions de hidrogênios $(\mathrm{pH})$ sozinho não é capaz de influenciar nos processos biocorrosivos; o potencial erosivo de um alimento; acidez titulável, cálcio, fosfato, níveis de flúor e outros fatores também devem ser considerados. Há muitos fatores biológicos 
intrínsecos interagindo na superfície dental, como qualidade de saliva e fatores pré-existentes de cada individuo como xerostomia, anorexia nervosa, bulimia e problemas gastroesofágicos. Evidencia-se então que há indivíduos que exibem mais biocorrosão do que outros, mesmo que sejam expostos exatamente ao mesmo desafio ácido em suas dietas. Portanto, a biocorrosão apresenta etiologia multifatorial, modulados por fatores químicos, biológicos e comportamentais ${ }^{12,15}$

As lesões se apresentam com um esmalte de brilho fosco, depressões rasas, polidas, lisas e largas, degaste de estrutura calcificada, localizada com mais frequência na superfície vestibular ou lingual, com degraus e concavidades, sem ângulos nítidos. Em regiões oclusais, as cúspides podem se encontrar arredondadas ou com concavidades até a dentina; e quando há a presença de material restaurador, este pode se encontrar com o limite elevado em relação á estrutura dental remanescente ${ }^{12}$.

\section{- Tratamentos}

$\mathrm{Na}$ literatura, varias formas de recursos terapêuticos são encontradas com a finalidade de minimizar a sintomatologia da HD. Estes, portanto devem ter inicio a partir de diagnostico clinico e diferencial que são muitas vezes negligenciados. É de suma importância que o profissional tenha conhecimento de fatores etiológicos associados à $\mathrm{HD}$, sem que haja sub ou superestimação desta condição ${ }^{2-16}$.

Considera-se que a orientação profissional juntamente com a colaboração do paciente em eliminar possíveis fatores etiológicos causais, estrutura-se base de inicio de terapia para minimizar os sintomas da HD, segundo a Divisão Canadense sobre Hipersensibilidade Dentinária ${ }^{2}$.

Os tratamentos para a hipersensibilidade podem ser classificados como de uso profissional e caseiro. $\mathrm{O}$ de uso profissional são produtos que demandam mais habilidade, pois são mais complexos e caros, apresentam-se na forma de géis ou aquoso e é mais empregado quando a dor é de maior grau. Já os de uso caseiro apresentam-se em forma de dentifrícios, enxaguatórios bucais entre outros e são mais fáceis de serem manipulados e apresentam menor custo ${ }^{2}$.

O mecanismo de ação das principais substancia denominadas dessensibilizantes, aqueles que tiram a exacerbação da sensibilidade, podem atuar de forma oclusiva e/ou neural; sendo os de método oclusivo os de principal emprego, estes podem atuar sobre os seguintes aspectos como: a alteração do conteúdo dos túbulos dentinários por coagulação, deposição de proteínas, formação e deposição de complexos de cálcio insolúveis e/ou recobrimento da dentina. Por sua vez, os agentes que apresentam ação neural agem despolarizando as membranas das fibras nervosas, bloqueando a transmissão de sinais dolorosos ao sistema nervoso central $^{2,17}$.

\section{- Dentifrícios}

A indicação de dentifrícios dessensibilizantes constitui ser uma das primeiras alternativas de indicação profissional para tratamento, devido seu método simples, fácil execução, e tendo como vantagem a aplicação feita em casa. Por obter composição muito complexa seu mecanismo de ação irá depender do tipo de agente dessensibilizante que contém. As principais substâncias que podem ser encontradas nos dentífricos para esta finalidade são os sais de potássio, a exemplo do cloreto, citrato e nitrato de potássio; cloreto de estrôncio, arginina e o hidróxido de cálcio. Sendo que a maioria desses compósitos como cloreto de estrôncio e hidróxido de cálcio, irá atuar de forma oclusiva sobre os túbulos dentinários através da deposição de cristais e fosfato de cálcio. Por sua vez o nitrato de potássio age na despolarização das fibras nervosas impedindo a passagem do estimulo doloroso para o sistema nervoso central. Estudos mostram que a arginina, um aminoácido natural presente na saliva, promove excelentes benefícios na terapia anti-hiperalgica. Determina-se que a arginina e o carbonato de cálcio ligam-se a superfície dentinária, carregando-a negativamente promovendo uma camada protetora rica em cálcio que irá impedir a transmissão de estímulos hidrostáticos. Apesar de estudos avaliarem grande vantagem da arginina como tratamento duradouro mesmo com a exposição frente a ácidos, em geral a matriz promovida pelos dentifrícios pode ter um tempo de ação de 2 a 12 semanas, constituindo esta uma possível desvantagem no emprego ${ }^{17-20}$.

\section{- Material restaurador}

As restaurações com cimento de ionômero de vidro e resina composta são indicadas quando além de hipersensibilidade dentinária relatada pelo paciente, as lesões cervicais não cariosas também apresentarem lesões cavitadas associadas, essas restaurações deveram ser realizadas após a remoção do fator causal ${ }^{19,21}$.

O cimento de ionômero de vidro como material restaurador possui a vantagem de ser hidrofílico e não requerer ataque ácido previamente. Tanto os comuns como os modificados, resinosos, mostram-se vantajosos em seu emprego com atuação na forma protetiva do tecido tubular exposto e na liberação de flúor no meio oral. Entretanto devido sua difícil inserção e por possuírem menor resistência ao desgaste, à compressão e à tensão, bem como propriedades ópticas inferiores, estes são menos utilizados comparados as resinas, considerando-se como tratamento alternativo. $\mathrm{O}$ tratamento restaurador em resina composta mostra-se importante recurso a ser aplicado na oclusão de tecido dentinario exposto $^{16,19-21}$. 


\section{○ Adesivos}

Os adesivos são indicados devido a facilidade no emprego da técnica e sua eficiência imediata. Seu principal mecanismo de ação é selar a entrada dos túbulos dentinário pela formação de camada hibrida, composta por dentina e pelo próprio adesivo. É indicado o emprego de adesivos autocondicionantes, de dois passos, devido sua desmineralização seletiva sem a formação de "gaps", áreas condicionadas sem receber o sistema adesivo ${ }^{16-19}$.

\section{- Fluor e verniz fluoretado}

Os fluoretos vêm sendo utilizados sobre diversas apresentações comerciais, podendo ser empregados tanto no tratamento relacionado a cárie dentária quanto em casos de HD. Podem estar contidos em dentifrícios, ou em vernizes, géis e soluções para bochecho. Constitui uma de suas vantagens o baixo custo; contudo, a aplicação deve ser feita por um cirurgião-dentista no consultório odontológico $^{2}$.

A aplicação de flúor tópico na forma de fluoreto de sódio é utilizada com o objetivo de obstrução dos túbulos dentinários expostos ao meio bucal; as substâncias fluoretadas quando entram em contato com a superfície mineralizada do dente reagem quimicamente com os íons de fosfato e cálcio e precipitam na forma de cristais de fluoreto de cálcio, resultando na oclusão dos túbulos. Porém os cristais formados dessa reação química possuem menor diâmetro que os túbulos dentinários, necessitando repetir a técnica até a completa obstrução do mesmo. Constitui uma de suas desvantagens a dissociação, por ser um composto instável o fluoreto de cálcio apresenta efeito de curta duração $^{17,22}$.

O verniz fluoretado mostra-se importante recurso relacionado a terapêutica para minimizar a sintomatologia em casos de HD. Seu mecanismo de ação mostra-se eficiente pela formação de película impermeabilizante, selando a superfície dentinária. Possui a vantagem de atuar como fonte de compostos fluoretados. Estudos demonstram o verniz fluoretado como mais eficiente quando comparado com adesivos e dentifrícios, sendo necessária uma única aplicação para a completa obliteração dos túbulos em todas as amostras. Para o adesivo, foram necessárias duas aplicações, e em relação ao dentifrício com dessensibilizante foram necessárias cinco aplicações. Entretanto, o efeito selante dos vernizes são de curta duração, já que a película é facilmente removida ${ }^{17,20}$.

\section{- Laser e novas terapêuticas}

Os lasers empregados atualmente como tratamento da hipersensibilidade dentinária, apresentam-se em duas modalidades: de baixa potência $\mathrm{HeNe}$, GaAlAs (diodo) e os de alta potência (CO2, Nd:YAG). Os laser de baixa potência tem ação moduladora, analgésica, antietémico e antiinflamatório das respostas celulares, possibilitando a liberação de endorfinas $\alpha$ e $\beta$ exógenas promovendo o bloqueio dos estímulos. Já os laser de alta potência atuam na desnaturação e coagulação de proteínas, causando a obliteração dos túbulos dentinários ${ }^{21,22}$.

O tratamento com o laser tem mostrado bastante eficiência, porém este não é um método definitivo, tendo que ser realizado várias aplicações para controle; além de ser uma técnica que demanda bastante conhecimento do profissional e alto custo para o paciente e para o profissional. Autores concluem que não há diferenças significativas entre os resultados obtidos entre os lasers de baixa e alta potencia. Demonstram um efeito positivo tanto no seu uso isolado quanto conjugado com outras propostas terapêuticas de maneira sinérgica e ao longo prazo ${ }^{2,11,16}$.

Vidros bioativos mesoporosos (MBG) são materiais inorgânicos, que vem demonstrando grande potencial de agente para o tratamento da HD. Apresenta-se como um material inorgânico, com características semelhantes a porção inorgânica da dentina. O MBG possui capacidade de diminuição de permeabilidade dentinaria quando associado ao acido fosfórico, resultando assim na dissolução e precipitação de di-hidrato de fosfato dicalcio. Produto este que recobre de forma homogênea a porção exposta, e evita a movimentação por estimulo externo do fluido intertubular, ocasionando, por conseguinte a mineralização e obliteração dos túbulos dentinarios regredindo a sintomatologia dolorosa ${ }^{2}$.

Novas áreas de pesquisa para o tratamento de HD vêm sendo exploradas, e o uso de agentes naturais mostram-se a base para novos estudos. A utilização da própolis em estudos comparativos, demonstraram sua eficiência comparada ao nitrato de potássio proporcionando tanto alivio imediato quanto ao final de 3 meses. Entretanto seu mecanismo de ação ainda é desconhecido, porém destaca-se que sua utilização de forma terapêutica contribui de forma significativa para oclusão tubular utilizada tanto de forma isolada como associada a outros agentes ${ }^{2}$.

DISCUSSÃO

A hipersensibilidade dentinária (HD), pode ser definida como o desconforto gerado frente a perda do material de proteção, o esmalte dental, e exposição dos túbulos dentinários devido a fatores químicos e mecânicos de baixa intensidade e alta frequência, sendo estes adquiridos, portanto não podendo ser associados a nenhuma outra patologia dental. Um dos assuntos mais comumente relacionados a esta alteração são as lesões cervicais não-cariosas (LCNCs) sendo elas: abrasão, abfração, atrição e erosão. A teoria hidrodinâmica de Brännström, é a mais aceita atualmente para explicar esse fenômeno relacionado a HD, devido a perturbação do fluido no interior dos túbulos dentinários ${ }^{1-6}$. Apesar da dificuldade em se obter uma 
metodologia sistemática para obtenção de dados sobre a prevalência da HD, estudos indicam que sua incidência vem aumentando quando comparada a estudos mais contemporâneos ${ }^{7-9}$. A história prévia da hipersensibilidade bem como a correlação de fatores como presença de LCNCs e predisposições a hábitos que afetem na sintomatologia direcionarão $o$ profissional ao correto diagnostico, planejamento e tratamento $^{10-15}$. Atualmente no cenário geral, os tratamentos buscam a obliteração dos túbulos dentinários sendo os materiais mais empregados, na forma de dentifrícios com agentes dessensibilizantes, aplicação tópica de flúor, adesivos e materiais restauradores ,porém há um grande desafio no emprego destes produtos, em questão relacionada a durabilidade e eficiência que devem ser levadas em considerações. Os estudos aplicados sobre de Lasers e novas terapêuticas vem ganhando espaço, e novos materiais naturais podem surgir ao longo do tempo com o mesmo objetivo ${ }^{16-22}$.

\section{CONCLUSÃO}

Com a expectativa de vida aumentando, um novo segmento de pacientes surge a procura de tratamento no âmbito odontológico e questões sobre HD, e LCNCs mostram-se em crescente evolução devido a maior tempo de exposição dos indivíduos. Diversos tratamentos mostram-se eficaz na redução da sintomatologia porém devido a suas características efêmeras, demonstram dificuldade na obtenção de métodos duradouros para esta aplicação. Faz se necessário a realização de novos estudos a cerca de lasers e novos métodos terapêuticos naturais em busca de vantagens a cerca do fator duração e estabilidade com a finalidade de se obter melhores indicações e mecanismos de tratamento.

\section{REFERÊNCIAS}

1. Douglas-De-Oliveira DW, Lages FS, Paiva SM, Cromley JG, Robinson PG ,Cota LOM. Crosscultural adaptation of the Brazilian version of the Dentine Hypersensitivity Experience Questionnaire (DHEQ-15). Braz. Oral Res. 2018;32-7

2. Silva MF, Perondi PR, Carvalho LR, De Araújo BN, De Carvalho CF, Quintella BLB et al. Hipersensibilidade dentinária: desafios para diagnósticos e perspectivas de tratamento. Rev Assoc Paul Cir Dent. 2017;71(2):170-74

3. Santos FFC, Lopes FF, Thomaz EBAF, Benatti BB, Pereira AFV. Avaliação de Lesões Cervicais Não-Cariosas em Adultos: Estudo Piloto. Pesq Bras Odontoped Clin Integr 2013;13(1):31-6

4. Pereira JC, Anauate-Netto C, Gonçalves SA. Complexo dentinho pulpar: fisiologia e resposta às injúrias. In: Dentística: uma abordagem multidisciplinar. São Paulo:Artes Médicas; 2014.

5. Chermont AB, Shimaoka AM, Andrade AP, Silva
CM, Carvalho RCR. O uso prévio de dessensibilizantes interfere na resistência de união de sistemas adesivos à dentina? Rev Assoc Paul Cir Dent. 2017;71(4):406-12

6. Machado AC, Matos AB, Rios LFF, Turbino ML, Scaramucci T. Hipersensibilidade dentinária: revisão de literatura e direcionamentos para abordagem clínica. Rev Assoc Paul Cir Dent. 2018;72(2):550-56

7. Zado NL, Pilatti LG. Hipersensibilidade dentinária: recentes avanços e tratamentosrevisão de literatura. Braz J Periodontol. 2016; 26(2):28-33

8. Scaramucci T, de Almeida Anfe TE, da Silva Ferreira S, Frias AC, Sobral MA. Investigation of the prevalence, clinical features, and risk factors of dentin hypersensitivity in a selected Brazilian population. Clin Oral Investig. 2014;18(2):651-57.

9. Fischer C, Fischer RG, Wennberg A. Prevalence and distribution of cervical dentine hypersensitivity in a population in Rio de Janeiro, Braz J Dent. 1992;20(5):272-76.

10. Marquezini Junior L, Sundfeld RH, Briso ALF, Mauro SJ, Okida RC. Hipersensibilidade dentinária em lesões cervicais com ou sem cavitação. JBD. 2002;1(3):245-54

11. Brentegani, LG, Lacerda, AS, Campos, AA. Alterações Regressivas dos Dentes: Abfração; Abrasão, Atrição e Erosão. Rev Bras Teleodonto. 2006;1(1):11-7

12. Xavier AFC, Pinto TCA, Cavalcanti AL. Lesões Cervicais não cariosas: um panorama atual. Rev Odontol Univ Cid. São Paulo 2012;24(1):57-66

13. Grippo JO, Simring M, Coleman TA. Abfraction, abrasion, biocorrosion, and the enigma of noncarious cervical lesions: a 20-year perspective. J Esthet Restor Dent. 2012;24(1):10-23.

14. Rios ACF, Lopes SCFL, Dantas TS, Oliveira VMB, Santos LB. ABRASIVOS :uma análise de dentifrícios comercializados em Salvador. Rev Bahiana Odontol. 2014;5(3):141-52

15. Lussi A. Erosive tooth wear - a multifactorial condition of growing concern and increasing knowledge. In: Dental Erosion. Monogr Oral Sci. Basel, Karger; 2006.

16. Tonetto MR, Dantas ABR, Bortolini GDF, Fabris M, Edson AC, Andrade MF. Hipersensibilidade dentinária cervical: em busca de um tratamento eficaz. Rev Odontol Univ Cid São Paulo 2012;24(3):190-99

17. Ribeiro PJT, Araújo AMP, Mafra RP, Vasconcelos MG, Vasconcelos RG. Mecanismos de ação dos recursos terapêuticos disponíveis para o tratamento da hipersensibilidade dentinária cervical. Odontol Clín Cient. 2016;15(2):83-90

18. Godinho CJ, Grippi MF, Costa LC. Avaliação clínica do uso de dois novos cremes dentais no tratamento da hipersensibilidade dentinária. RPG 
Rev Pós Grad.2011;18(2):72-8

19. Aguiar FHB, Giovanni EM, Monteiro FHL, Villalba H, Mello JJ de, Tortamano N. Hipersensibilidade dentinária- causas e tratamentos. Uma revisão da literatura.Rev Ins Ciênc Saúde.2005; 23(1):67-71

20. Rocha CS, Prado M, Simão RA, Lima CO, Gusman H. Efeito de agentes dessensibilizantes na obliteração dos túbulos dentinários - estudo in vitro. Rev bras odontol. 2016;73(4):272-76.

21. Querido MTA, Raslan AS, Scherma AP. Hipersensibilidade dentinária - revisão da literatura. Periodontia 2010;20(2):39-46

22. Mesquitas CN, Almeida JCF, Yamagutic PM, De Paula LM, Garcia FCP. Hiperestesia dentinária: opções de tratamento. Dentística on line. 2009;8(18):29-34

\section{CONFLITO DE INTERESSES}

Os autores declaram não haver conflitos de interesse.

\section{AUTOR PARA CORRESPONDÊNCIA}

\section{Heloise Nogueira Rocha}

E-mail: heloise-rocha@hotmail.com 